\title{
Misdiagnosis of solid pseudopapillary neoplasm - a case report
}

Francesca De Stefano ${ }^{1}$, Antonella D'Addiego ${ }^{2}$, Ottavia Caserini ${ }^{1}$, Giorgio M.P. Graziano ${ }^{1}$, Alessandro Vanoli ${ }^{3,4}$, Emanuela Boveri ${ }^{3,4}$, Riccardo Carbone ${ }^{3,4}$, Marco Paulli ${ }^{3,4}$ and Tommaso Manciulli ${ }^{1}$, Marcello Maestri ${ }^{2 *}$

${ }^{1}$ Dipartimento di Scienze clinico-chirurgiche, diagnostiche e pediatriche, Università di Pavia, Italy

${ }^{2}$ Dipartimento di Chirurgia, S.C. Chirurgia Generale I, Fondazione IRCCS Policlinico San Matteo, Pavia, Italy

${ }^{3}$ Dipartimento di Medicina Molecolare, Università di Pavia, Italy

${ }^{4}$ S.C. di Anatomia Patologica, Fondazione IRCCS Policlinico San Matteo, Pavia, Italy

\begin{abstract}
Background: Solid pseudopapillary neoplasm of the pancreas is a rare epithelial tumor. It represents $1 \%$ out of $3 \%$ of all exocrine pancreatic tumors. It commonly affects young women. The clinical presentation is not specific and radiological examinations are often non diagnostic. CT scan seems the most appropriate diagnostic tool, but presentations can vary. The treatment of choice is the surgical resection and the prognosis is usually favorable.

Case presentation: An 81-year-old woman with a previous diagnosis of hydatid cyst of the pancreatic tail was referred to our Institute due to the onset of a retroperitoneal lesion at CT scan. Radiological features of the retroperitoneal mass were suspicious for sarcoma. The multidisciplinary committee proposed a surgical exploration for a definitive diagnosis. Intraoperatively, the mass appeared not to be of retroperitoneal origin, but it was strictly attached to the previously known cyst in the pancreatic tail. The patient underwent a distal splenopancreasectomy. At the histological examination both the cyst and the mass of the pancreatic tail were solid pseudopapillary neoplasms.

Conclusion: In this clinical case, misleading clinical and radiological features as well as the poor experience in SPN diagnosis did not allow a correct preoperative workup. Only the postoperative histological examination led to the correct diagnosis of both the long-term pancreatic cyst and the adjacent mass. Our experience highlights the importance of differential diagnosis of atypical pancreatic lesions, among which SPN should always be included.
\end{abstract}

Abbreviations: Solid pseudopapillary tumor (SPN); Magnetic resonance imaging (MRI); Computed tomography (CT); Ultrasound (US); Neuron specific enolase (NSE).

\section{Background}

Solid pseudopapillary tumor (SPN) of the pancreas is a pancreatic neoplasm with low-grade malignant potential. SPN is a quite rare entity, accounting for up to $3 \%$ of pancreatic tumors and overall, $5 \%$ of pancreatic cystic lesions. It predominantly affects young women, with average age of onset being 22 years [1,2]. Body and tail of the pancreas are mainly involved while the head is an uncommon localization. Computed tomography (CT) and magnetic resonance imaging (MRI) are important tools for preoperative diagnosis and allow to study relationships of the lesion with the adjacent structures [3]. SPN is usually large and encapsulated, with a mixture of cystic, solid, and hemorrhagic components [2]. The clinical presentation is not specific and radiological examinations are often non diagnostic, therefore SPN is often misdiagnosed [4]. The prognosis is generally favorable when surgical resection with negative margins is performed [5].

\section{Case presentation}

An 81-year-old Caucasian woman was in follow-up for a hydatid cyst located at the tail of the pancreas at a community hospital. The diagnosis was made in 2009 on the ground of CT abdomen describing a $10 \mathrm{~cm}$ subdiaphragmatic mass in the left upper quadrant surrounded by an outer calcific profile with inner septa and corpuscolated material.
Serology and Western-Blot were both negative for echinococcosis. Nevertheless, the long-term stability of the cyst during follow-up, the radiological features as well as the geographical origin of the patient, were all compatible with the initial diagnosis. The abdominal ultrasound (US) performed in 2019 during follow-up assessed the stability of the hydatic cyst $(89 \times 90 \mathrm{~mm}$, WHO classification type CE5). However, it also showed the onset of a mass adjacent to the cyst in the left upper retroperitoneal space. Because of the new US finding, the patient was referred to our center.

Her previous medical history was characterized by hypereosinophilic syndrome, monoclonal gammopathy, rheumatoid arthritis, hysterectomy for uterine leiomyoma and appendectomy. Physical examination did not reveal any abnormal finding. Laboratory analysis indicated a rise of blood concentration of amylase $(262 \mathrm{mU} / \mathrm{ml})$ and bilirubin $(1.70 \mathrm{mg} / \mathrm{dl})$, leukocytosis $(12.6 \mathrm{x} 103 / \mathrm{ul})$ and mild anemia (hemoglobin: $9.2 \mathrm{~g} / \mathrm{dl}$ ). At the abdominal CT performed at our Institute, the mass (diameters $55 \times 39 \mathrm{~mm}$ ) was apparently originating from the

*Correspondence to: Marcello Maestri, Dipartimento di Scienze clinicochirurgiche, diagnostiche e pediatriche, Università di Pavia, Italy, E-mail: mmaestri@smatteo.pv.it

Key words: pancreas, epithelial tumors, echinococcosis, hydatid cyst, pancreatic surgery, solid pseudopapillary neoplasm

Received: September 07, 2020; Accepted: September 18, 2020; Published: September 21, 2020 
retroperitoneum and it was located behind the upper pole of the left kidney and the left psoas muscle (Figure 1). The mass had a proper vascularization, and despite the lack of infiltration, it had no cleavage plane with the left kidney, the left psoas muscle and the posterior side of the hydatid cyst (Figure 2,3). Considering the radiological features, a sarcoma or a fibromatous lesion were hypothesized.

After a multidisciplinary panel discussion, the patient was candidated to surgery and an explorative laparotomy was performed. Intraoperatively, the mass appeared within the pancreatic tail, strictly attached to the hydatid cyst and the splenic vessels. A cleavage plane with the left kidney and the psoas muscle could be detected and thus a distal splenopancreatectomy was performed, including both the pancreatic mass and cyst.

Histological examination of the two contiguous pancreatic lesions (the cyst and the solid mass) was diagnostic for pancreatic solid pseudopapillary neoplasms (Figure 4). No echinococcal infection was present in the specimen. Both lesions were delimited by a fibroconnective calcified capsule, focally windowed with overgrowth of tissue in the surroundings. Resection margins were negative (R0) without vascular or perineural invasion. The tumor cells were uniform, with bland and sometimes grooved nuclei, they were arranged in nests and cords, while pseudo-papillae and pseudo-cystic areas could be focally found (Figure 5). The immuno-histochemical profile was positive for vimentin,

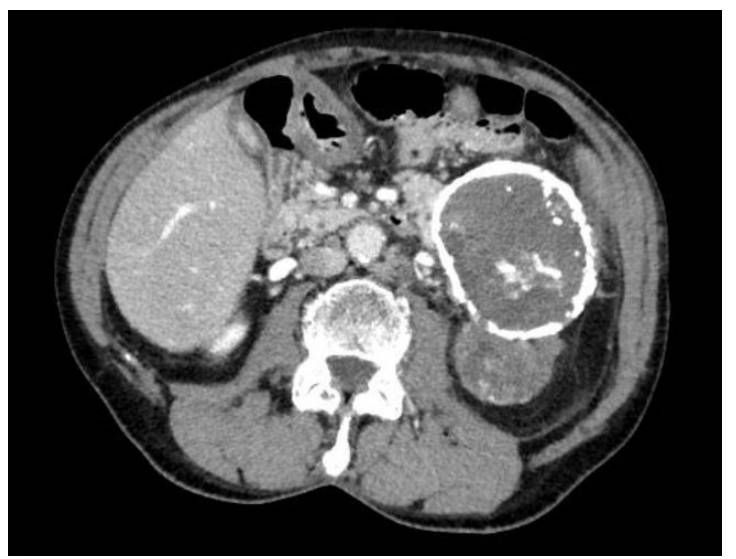

Figure 1. Axial $\mathrm{CT}$ scan of the abdomen demonstrating a large calcific mass arising from the tail of pancreas* and a retroperito-neal non-calcific mass

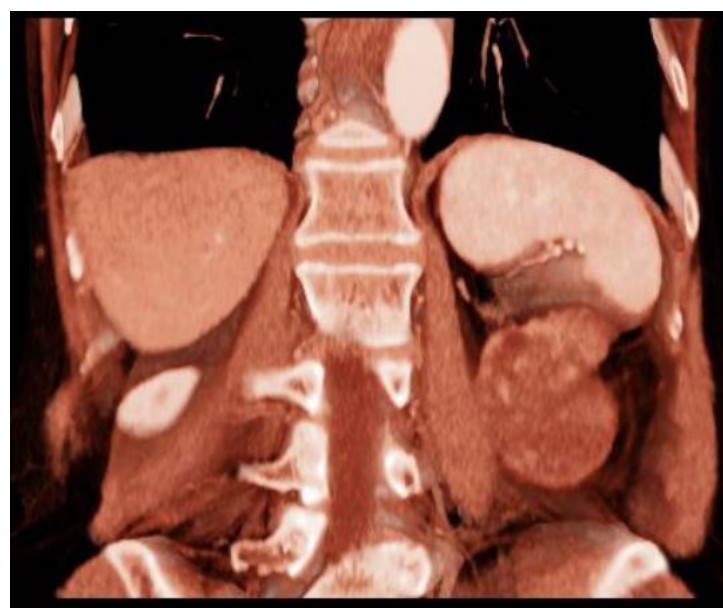

Figure 2. Coronal CT scan reconstruction

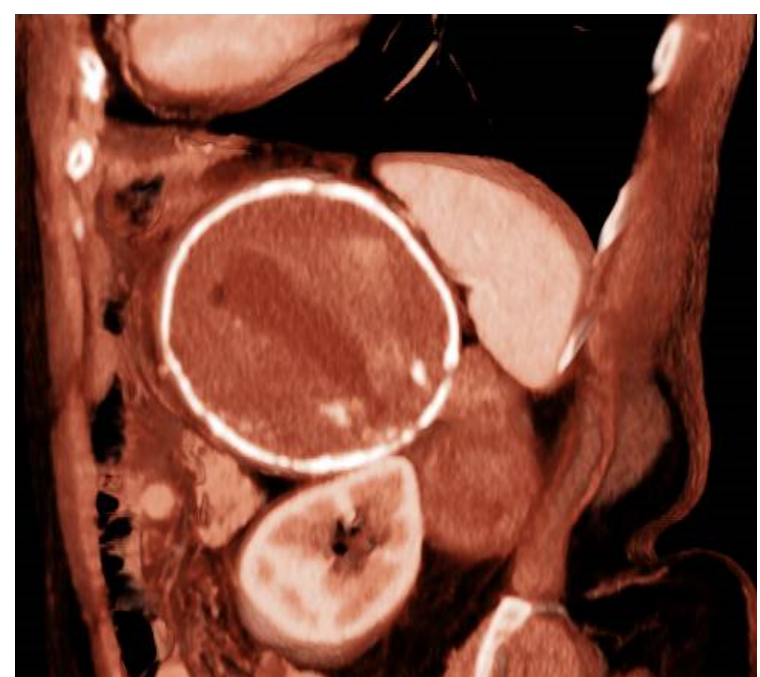

Figure 3. Sagittal CT scan reconstruction

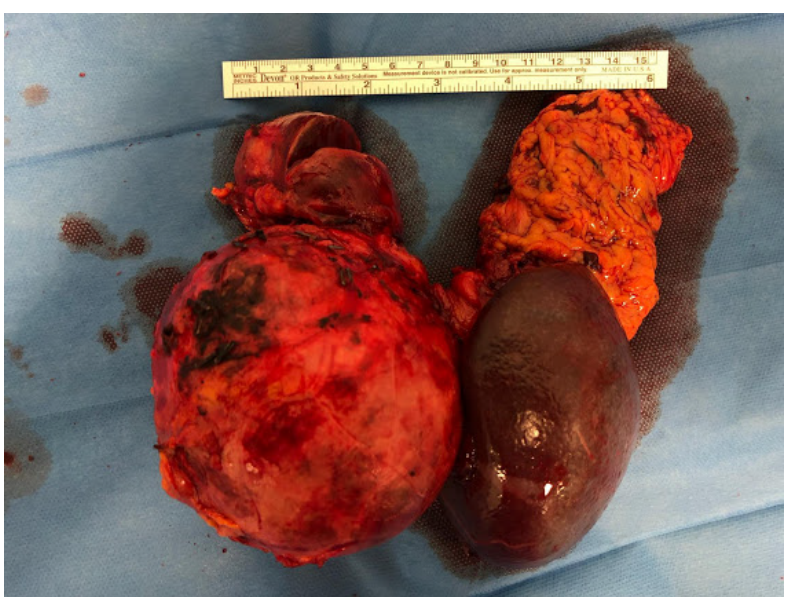

Figure 4. Surgical specimen demonstrating two large mass of the tail of pancreas involving spleen vessels

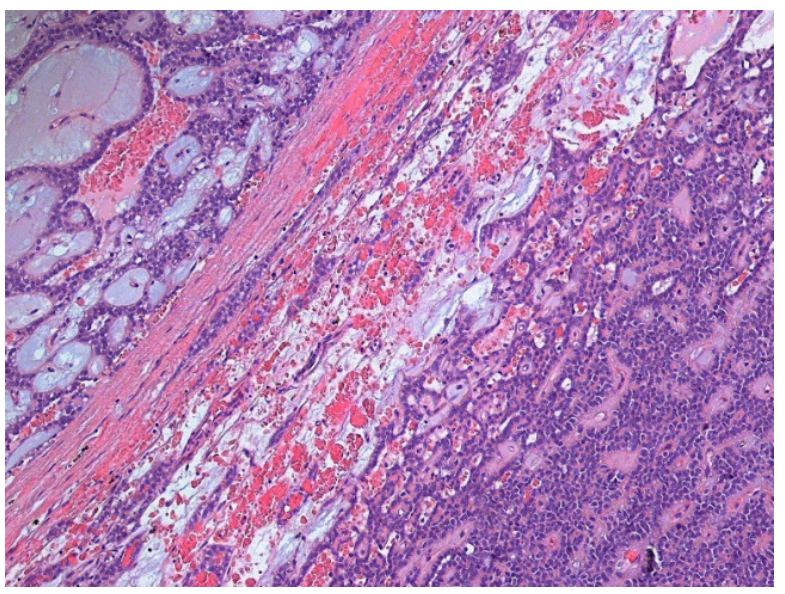

Figure 5. Histologic features of the solid pseudopapillary neoplasm (hematoxylin and eosin)

CD10, progesterone receptors, beta-catenin and partially positive to CD56, whereas it was negative for estrogen receptors, cytokeratin-7, synaptophysin, neuron specific enolase (NSE), chromogranin A. The Ki67 proliferative index was 5\% (Figure 6). 


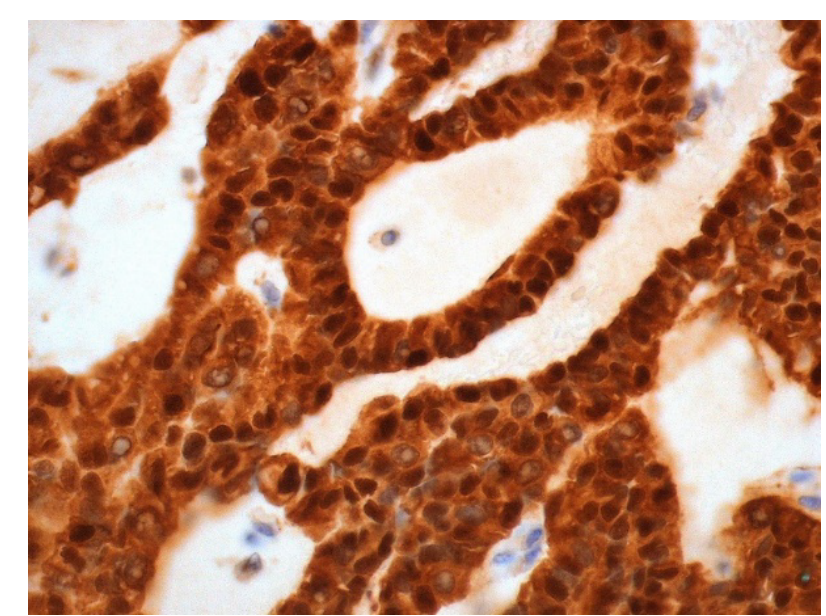

Figure 6. Nuclear expression of beta-catenin by the tumor cells (beta-catenin immunostaning)

The post-operative course was uneventful, and the patient was discharged 10 days after surgery. Considering the negative resection margins and the good conditions of the patient, no further therapies were indicated but oncological follow-up.

\section{Discussion}

SPN of the pancreas was first described in the literature by Frantz, in 1959. It is a rare exocrine pancreatic tumor with a low malignant potential. It represents around $5 \%$ of all cystic pancreatic neoplasms. The female to male ratio is of 9.7:1. According to the literature, SPN predominantly affects young women in the second or third decade of life, with an average age of onset being 22 years [1,2]. While the average patient age in extant studies, our patient was 81 years old. This notable age gap represented one of the reasons that led us not to consider SPN among the differential diagnoses in the preoperative workup.

At the macroscopic examination of the surgical specimen, the lesion appears of variable size and well encapsulated, containing necrosis, hemorrhagic areas and cystic zones. At the microscopic analysis, both solid and pseudopapillary arrangements can be identified [6]. SPNs have different characteristic patterns depending on tumor size: smaller tumors frequently are arranged in solid sheets, while larger lesions have a pseudopapillary pattern with residual tumor cells forming perivascular rosettes [7]. Although the pathogenesis of the tumor remains unclear $[4,8]$, the common nuclear accumulation of beta-catenin suggests a role of a mutation in the Wnt/beta-catenin signaling pathway in tumor onset $[9,10]$. In the presented case, the histopathological features of the surgical specimen were specific for the diagnosis of SPN.

The clinical presentation is variable, patients can be totally asymptomatic and SPN are incidentally discovered in about $25 \%$ of patients. Otherwise, patients may complain about non-specific symptoms like abdominal discomfort or pain due the dimension of the mass, back pain, nausea, vomiting and weight loss [1]. In our case report, the patient presented mild hyperbilirubinemia and hyperamylasemia without any clinical symptom.

Usually, SPN has a favorable prognosis with a 5-year survival rate of $97 \%$ and a 5 -year disease-free survival rate of $95 \%$ after complete surgical resection. SPN usually grows adjacent to other structures without infiltrating them $(5,6)$, as in our case. In 10 to $15 \%$ of cases it may assume an aggressive behavior, although the prognosis remains favorable [11]. The most relevant risk factors involved in the aggressive behavior of SPN are the incomplete capsule of the tumor, the infiltration into peripancreatic fat tissue, the involvement of lymph nodes, the perineural or vascular invasion and the tumor size [2]. Moreover, some studies reported that more active tumors may have a Ki67 index greater than $3 \%[12]$.

Diagnosis could be difficult and blood analysis or neoplastic markers are non-specific. According to the literature, CT scan is the most relevant examination for a correct diagnosis [3]. At CT scan, SPN appears as a heterogeneous mass, usually encapsulated, with cystic and solid components mixed in different percentages. On enhanced CT images the solid part of the tumor appears hypodense with respect to the normal parenchyma in arterial and delayed phase, while it has a progressive enhancement in venous phase [2]. The lesion is usually delimited by a peripheral capsule made of fibroconnective tissue, but it has been reported also the presence of a calcified shell that surrounds the neoplasm [13]. In the clinical case presented, the solid mass had an unusual presentation at imaging, because no calcific component was detectable at the $\mathrm{CT}$ scan, making the preoperative diagnosis more challenging.

Abdominal endoscopic-US can be useful in tumor staging and surgery planning, because it provides details on pancreatic or peripancreatic lesions, tumor size, vascular invasion and metastasis [3]. Even though imaging is paramount in preoperative workup, histological data may be useful for the differential diagnosis with other pancreatic cystic lesions. Endoscopic US-guided biopsy (EUS-FNA) has high sensitivity (91\%) and specificity (94\%) and low risk of neoplastic dissemination, thus it could be considered as the most effective tool. Percutaneous US- or CT-guided core needle biopsy implies a higher risk of dissemination [14]. In our clinical case, the US features of the pancreatic cyst were compatible with CE5 hydatid cyst. In addition, the solid mass was erroneously considered a retroperitoneal tumor and the pancreatic origin was not suspected. Due to these reasons, endoscopicUS or EUS-FNA were not included in the preoperative workup.

Surgery is the treatment of choice for SPN. An organ-preserving resection is the usual choice due to low-grade malignant potential of SPN. Distal pancreatectomy with or without splenectomy, pancreaticoduodenectomy or total pancreatectomy are usually performed depending on the location of the tumor. A recent study demonstrated that there is no significant difference between the open and the laparoscopic approach in terms of recurrence or overall survival and that laparoscopic technique is not related to a higher risk of dissemination [15]. The most relevant prognostic factor is the radicality of the resection. An R0 en-bloc resection is the fundamental parameter improving overall survival rate and disease-free survival rate [12]. Lymphadenectomy is not usually performed, unless there is a strong evidence of lymph node involvement, due to the rarity of lymph node metastasis. Local recurrence rate is lower than $10 \%$ and it is usually reserved to patients with metastases or an unresectable mass at diagnosis [16]. The management of these cases is still under debate due to the favorable prognosis and the rarity of aggressive SPN reported in literature. Indeed, different strategies have been used like adjuvant chemotherapy, neoadjuvant chemotherapy for unresectable metastasis, aggressive surgical approaches with surgical excision of metastasis and even HIPEC, but until now no consensus has been reached on the best therapy [12]. Further studies are needed to define the best approach in these uncommon cases.

Our patient underwent surgical exploration in the suspect of retroperitoneal sarcoma. Considering the intraoperative findings, a 
distal splenopancreasectomy was performed. The resection was R0, therefore the patient was properly treated for SPN. No further therapies were necessary, she was addressed to oncological follow-up and her prognosis was favorable. At 1-year follow-up the patient is alive and disease-free.

\section{Conclusions}

The case report presented in this manuscript highlights the possible challenges encountered in the radiological preoperative diagnosis of SPN, due to its low incidence and often non-typical findings. Limitations in our experience were mainly due to the age of the patient and the misleading initial diagnosis of hydatic cyst. A more accurate diagnostic workup should always be performed in case of atypical pancreatic lesions. In particular, SPN has to be considered among the differential diagnoses in these cases. However, the definitive diagnosis is sometimes reachable only after surgical resection.

\section{Declarations}

\section{Ethics approval and consent to participate}

Ethics approval for the study has been obtained.

\section{Consent for publication}

Written consent was obtained from the patient for publication of study.

\section{Availability of data and materials}

Not applicable.

\section{Competing interests}

The authors declare that they have no competing interests.

\section{Funding}

No funds were obtained for this study.

\section{Acknowledgement}

Not applicable.

\section{Authors' contributions}

- Francesca De Stefano: collection of the data, revision of the literature and writing of the manuscript.

- Antonella D’Addiego, Ottavia Caserini, Giorgio M.P. Graziano: they equally collaborated in the writing and revision processing of the manuscript and revision of the literature.

- Alessandro Vanoli, Emanuela Boveri, Riccardo Carbone, Marco Paulli: they equally collaborated in the histological examination after surgery and in the revision of the manuscript.
- Marcello Maestri: supervision of the research, surgical procedure, revision of the results.

\section{References}

1. Papavramidis T, Papavramidis S (2005) Solid pseudopapillary tumors of the pancreas: review of 718 patients reported in English literature. J Am Coll Surg 200: 965-972. [Crossref]

2. Estrella JS, Li L, Rashid A, Wang H, Katz MH, et al (2014) Solid pseudopapillary neoplasm of the pancreas: clinicopathologic and survival analyses of 64 cases from a single institution. Am J Surg Pathol 38: 147-157. [Crossref]

3. Del Chiaro M, Verbeke C, Salvia R, Klöppel G, Werner J, et al (2012) European experts consensus statement on cystic tumours of the pancreas. Dig Liver Dis 45: 703-711. [Crossref]

4. Naar L, Spanomichou DA, Mastoraki A, Smyrniotis V, Arkadopoulos N (2017) Solid Pseudopapillary Neoplasms of the Pancreas: A Surgical and Genetic Enigma. World $J$ Surg 41: 1871-1881. [Crossref]

5. Lubezky N, Papoulas M, Lessing Y, Gitstein G, Brazowski E, et al (2017) Solid pseudopapillary neoplasm of the pancreas: Management and long-term outcome. Eur J Surg Oncol 43: 1056-1060. [Crossref]

6. Reddy S, Cameron JL, Scudiere J, Hruban RH, Fishman EK, et al (2009) Surgica management of solid-pseudopapillary neoplasms of the pancreas (Franz or Hamoudi tumors): a large single-institutional series. J Am Coll Surg 208: 950-957. [Crossref]

7. Klimstra DS, Wenig BM, Heffess CS (2000) Solid-pseudopapillary tumor of the pancreas: a typically cystic carcinoma of low malignant potential. Semin Diagn Pathol 17: 66-80. [Crossref]

8. Kosmahl M, Seada LS, Jänig U, Harms D, Klöppel G (2000) Solid-pseudopapillary tumor of the pancreas: its origin revisited. Virchows Arch 436: 473-480. [Crossref]

9. Tanaka Y, Kato K, Notohara K, Hojo H, Ijiri R, et al. (2001) Frequent beta-catenin mutation and cytoplasmic/nuclear accumulation in pancreatic solid-pseudopapillary neoplasm. Cancer Res 61: 8401-8404. [Crossref]

10. Abraham SC, Klimstra DS, Wilentz RE, Yeo CJ, Conlon K, et al (2002) Solidpseudopapillary tumors of the pancreas are genetically distinct from pancreatic ductal adenocarcinomas and almost always harbor beta-catenin mutations. Am J Pathol 160 1361-1369. [Crossref]

11. Antoniou EA, Damaskos C, Garmpis N, Salakos C, Margonis GA, et al (2017) Solid Pseudopapillary Tumor of the Pancreas: A Single-center Experience and Review of the Literature. In Vivo 31: 501-510. [Crossref]

12. Hao EIU, Hwang HK, Yoon DS, Lee WJ, Kang CM (2018) Aggressiveness of solid pseudopapillary neoplasm of the pancreas: A literature review and meta-analysis. Medicine 97: e13147. [Crossref]

13. Choi JY, Kim MJ, Kim JH, Kim SH, Lim JS, et al. (2006) Solid pseudopapillary tumor of the pancreas: typical and atypical manifestations. AJR Am J Roentgenol 187: W17886. [Crossref]

14. Petrakis I, Vrachassotakis N, Kogerakis N, Hatzidakis A, Zoras O, et al. (2001) Solid pseudopapillary neoplasm of the pancreas: report of a case after a 10-year follow-up and review of the literature. Pancreatology 1: 123-128.

15. Zhang RC, Yan JF, Xu XW, Chen K, Ajoodhea H, et al. (2013) Laparoscopic vs open distal pancreatectomy for solid pseudopapillary tumor of the pancreas. World $J$ Gastroenterol 19: 6272-6277. [Crossref]

16. Ren Z, Zhang P, Zhang X, Liu B (2014) Solid pseudopapillary neoplasms of the pancreas: clinicopathologic features and surgical treatment of 19 cases. Int J Clin Exp Pathol 7: 6889-6897. [Crossref]

Copyright: (C2020 De Stefano F. This is an open-access article distributed under the terms of the Creative Commons Attribution License, which permits unrestricted use, distribution, and reproduction in any medium, provided the original author and source are credited. 